\title{
EFECTO DEL TIPO Y TIEMPO DE FERMENTACIÓN EN LA CALIDAD FÍSICA Y QUÍMICA DEL CACAO (Theobroma cacao L.) TIPO NACIONAL
}

\section{EFFECT OF TIME AND TYPE OF FERMENTATION IN PHYSICAL AND CHEMICAL QUALITY OF COCOA (Theobroma cacao L.) NATIONAL TYPE}

\author{
${ }^{\bullet}$ Rubén Darío Rivera Fernández ${ }^{1,2}$, Freddy Wilberto Mecías Gallo², Ángel Monserrate Guzmán Cedeño ${ }^{1,2}$, Mayra Mercedes \\ Peña Galeas ${ }^{3}$, Hugo Nolti Medina Quinteros ${ }^{3}$, Lola Margarita Casanova Ferrín ${ }^{4}$, Alexandra Elizabeth Barrera Álvarez $z^{4}$, \\ Pedro Eduardo Nivela Morante ${ }^{3}$ \\ ${ }^{1}$ Jefatura de Investigación, Escuela Superior Politécnica Agropecuaria de Manabi Manuel Félix López. \\ Calceta, Manabi, Ecuador. \\ rd_03rivera@hotmail.com \\ ${ }^{2}$ Carrera de Agrícola, Escuela Superior Politécnica Ágropecuaria de Manabi Manuel Félix López. Calceta, Manabi, Ecuador. \\ ${ }^{3}$ Facultad de Ciencias Pecuarias, Universidad Técnica Estatal de Quevedo, $\mathrm{km} 7$ vía Quevedo - El Empalme, \\ C. P. 73. Mocache, Los Ríos, Ecuador. \\ ${ }^{4}$ Unidad de Investigación Científica y Tecnológica, Universidad Técnica Estatal de Quevedo, km 7 vía Quevedo - El Empalme, \\ C. P. 73. Mocache, Los Ríos, Ecuador.
}

\section{RESUMEN}

L a presente investigación tuvo como objetivo evaluar los tipos de fermentadores y los diferentes tiempos de fermentación usados por los productores de cacao de la zona norte y central de la provincia de ManabíEcuador. Se estudiaron cuatro fermentadores: saco de yute, montón, caja de madera y tina plástica, con una capacidad para almacenar $60 \mathrm{~kg}$ de cacao y tiempos de fermentación de 2 a 5 días; además, se evaluó un testigo (sin fermentar). El diseño fue bloques completos al azar en arreglo factorial aditivo con tres réplicas. Los datos se analizaron mediante análisis de varianza con la prueba de Tukey al 5\% de probabilidad. Se analizaron variables físicas: porcentaje de granos fermentados, violeta y pizarrosos (prueba de corte); y químicas: polifenoles (fotometría $760 \mathrm{~nm}$ ), acidez volátil (arrastre de vapor) y teobromina-cafeína (Cromatografía Líquida de Alta resolución, HPLC). El análisis de los resultados muestra que el tiempo de fermentación a diferencia del tipo de fermentador, tuvo influencia sobre las variables físicas y químicas analizadas. El aumento del porcentaje de granos fermentados y la disminución de los granos violetas ocurrieron desde el inicio hasta el final de la fermentación. La calidad química mejora con la reducción de la concentración de compuestos alcaloides y polifenoles, lo que repercute sobre las características organolépticas del cacao tipo Nacional. De los fermentadores y los tiempos utilizados por los productores se presentan las mejores características de calidad entre los cuatro y cinco días de fermentación en cajas de madera.

Palabras clave: Fermentadores, fermentación, polifenoles, alcaloides, acidez volátil, calidad del cacao.

Recibido: 8-Noviembre-2011. Recibido en forma corregida: 15-Junio-2012. Aceptado: 20-Junio-2012.

Publicado como ARTÍCULO CIENTÍFICO en Ciencia y Tecnología 5(1): 7-12. 2012

\section{Abstract}

T his investigation had as an objective to evaluate types of fermenters and different times of fermentation used by cocoa producers from the central and north zone of Manabi province, Ecuador. Four fermenters were used: burlap sack, piles, wooden boxes and plastic vat with the ability to store 60 kilos of cocoa and fermentation times ranging from 2 to 5 days; a control was evaluated in addition (without fermentation). A Completely Randomized Blocks Design with additive factors arrangement with three replications was used. The data were analyzed using analysis of variance with Tukey test at 5\% probability. Physical variables were analyzed: percentage of fermented, violet and slate (test cutting) grains; and chemical: polyphenols (photometry $760 \mathrm{~nm}$ ), volatile acidity (steam stripping) and theobromine, caffeine (High Performance Liquid Chromatography, HPLC). The analysis of the results shows that the fermentation time unlike the type of fermenter, had an influence on the chemical and physical variables analyzed. The increased percentage of fermented beans and decreased violet grains occurred from the beginning until the end of fermentation. The chemical quality improves by reducing the concentration of alkaloid compounds and polyphenols, which impacts on the sensory characteristics of Nacional cocoa type. Of the fermenters and the times used by producers the best quality characteristics are presented between four and five days of fermentation in wooden boxes.

Key words: Fermenters, fermentation, polyphenols, alkaloids, volatile acidity, cocoa quality. 


\section{INTRODUCCIÓN}

$\mathrm{A}$ ctualmente el cultivo de cacao (Theobroma cacao L.), representa uno de los rubros de mayor importancia económica para el Ecuador, ocupando el tercer lugar de las exportaciones no petroleras en el 2009 (Banco Central, 2010). Además, el país produce cacao de calidad única en el mundo, debido a sus características organolépticas, sin embargo en las últimas décadas estas características han sido afectadas debido al mal manejo post-cosecha (Enríquez, 2004).

La fermentación del cacao es una etapa muy importante en el procesamiento del grano, ya que se producen cambios bioquímicos que dan origen a los precursores del aroma y sabor (Contreras et al., 2002), lo que determina su calidad física y química (Manual de productos básicos, 1991).

Entre los cambios bioquímicos está el desarrollo de la pigmentación color marrón a partir de compuestos fenólicos (Cros y Jeanjean, 1995), lo cual es un indicativo de la fermentación del grano de cacao. Además, los contenidos de precursores sensoriales como polifenoles (Luna et al., 2002) alcaloides (cafeína y teobromina) (Bucheli et al., 2001) y acidez volátil (en especial el ácido acético) (Hill y Kold, 1999), son indicadores de la calidad organoléptica del cacao.

En la práctica, los métodos de fermentación varían mucho de una zona productora a otra, sobretodo el tipo de fermentador (Graziani de Fariñas et al., 2003) y el tiempo de fermentación, siendo los más usuales, entre los productores de la zona norte y central de Manabí, las cajas de madera, saco de yute, tinas plásticas y montón. Por su parte los tiempos de fermentación varían entre dos a cuatro días.

El objetivo del estudio consistió en evaluar los tipos de fermentadores y los diferentes tiempos de fermentación usados por los productores de zona norte y central de la provincia de Manabí-Ecuador, con la finalidad de establecer parámetros adecuados que mejoren la calidad del cacao ecuatoriano a nivel de productores y por ende los precios del producto.

\section{Materiales y MÉTOdos}

$\mathrm{S}_{\mathrm{d} d \mathrm{at}}^{\mathrm{e}}$ seleccionaron tres parcelas (una por cada localidad) de las localidades: Río Chico, Ricaurte y Calceta de la provincia de Manabí-Ecuador. Las parcelas contenían cacao de tipo Nacional, clonal, de entre 10 a 20 años de edad y como mínimo dos hectáreas del cultivo en producción. Los tratamientos fueron la combinación de los niveles de los factores: fermentadores (sacos de cabuya, montón, cajas de madera y tina plástica) con capacidad de $60 \mathrm{~kg}$ de cacao y tiempo de fermentación (dos, tres, cuatro y cinco días). Se utilizó un diseño de bloques al azar en arreglos factorial aditivo $(\mathrm{AxB}+1)$ con tres réplicas. El testigo consistió en un volumen de cacao similar al tamaño de los tratamientos pero sin ser sometido al proceso de fermentación. Los datos se analizaron mediante el análisis de varianza y para la comparación de medias de los tratamientos se empleó la prueba de Tukey al 5\% de probabilidad.

\section{Variables físicas}

Los parámetros físicos analizados fueron: porcentajes de granos fermentados, granos violetas y granos pizarrosos. Estos se evaluaron mediante la prueba de corte (Tomlins et al., 1993) que consistió en analizar la coloración interna del grano, así como las estrías que se forman producto de la fermentación, realizando cortes longitudinales a 100 granos secos con la finalidad de agrupar aquellos que presenten las siguientes similitudes:

Granos fermentados. Granos cuyos cotiledones presentan en su totalidad una coloración marrón o marrón rojiza y estrías profundas de fermentación.

Granos violetas. Granos cuyos cotiledones presentan una coloración violeta intenso.

Granos pizarrosos. Granos cuyos cotiledones presentan una coloración gris negruzco o verdoso y aspecto compacto.

\section{Variables químicas}

Fueron analizados los polifenoles, la acidez volátil y los alcaloides (teobromina y cafeína) de los granos de cacao. Los polifenoles se analizaron mediante la metodología de Recalde (2007), utilizando espectrofotometría a $760 \mathrm{~nm}$, y los datos se expresaron en mg de ácido gálico $\mathrm{g}^{-1}$ muestra. La determinación de acidez volátil se realizó por arrastre de vapor (Armijos, 2002), que consiste en remover los ácidos con ácido sulfúrico concentrado y luego destilarlos mediante el equipo Lickens Nickelson y finalmente titularlos con hidróxido de sodio $0.1 \mathrm{~N}$, usando como indicador fenolftaleína.

El análisis de teobromina y cafeína se realizó extrayendo en medio acuoso los alcaloides, los cuales fueron separados, identificados y cuantificados a través de un sistema de Cromatografía Líquida de Alta resolución, provisto de una columna de fase reversa C18UDS II (150 x $4.6 \mathrm{~mm}$ ID), precolumna del mismo material $(10 \times 4.6 \mathrm{~mm}$ ID) y de un detector UV/VIS a 273 nm (Espin et al., 2007). 


\section{Resultados y Discusión}

\section{Variables físicas}

$\mathrm{L}$ as variables físicas analizadas de los granos de cacao, se vieron influenciadas por los factores en estudio. Las reacciones bioquímicas, producto de la fermentación, provocaron que la coloración en el interior de los granos cambie progresivamente de violeta a marrón. El testigo (sin fermentar) mostró altos niveles de granos violeta, los que al fermentar el cacao, van disminuyendo a medida que avanza el tiempo de fermentación (Barel, 1987), alcanzando 75\% de coloración marrón en el quinto día de fermentación (Figura 1), al contrario, Graziani de Fariñas et al. (2003) obtuvieron $93.3 \%$ en igual tiempo de fermentación. Esta discrepancia podría radicar en la cantidad de la masa en fermentación $(3 \mathrm{~kg})$ usada por dichos autores, lo cual es un factor influyente en la fermentación (Puziah et al., 1998). Hasta el tercer día de fermentación se observó un incremento considerable de granos fermentados con respecto al testigo y es entre el cuarto y quinto día, cuando se obtuvo mayor tendencia en los cambios de coloración interna del grano, de violeta a marrón, los cuales son indicativos de un grano bien fermentado, que ha adquirido los precursores de sabor y aroma. Cros et al. (1982) mencionan que entre el segundo y cuarto día ocurre la inversión de la relación antocianinas monómeros polímeros ${ }^{-1}$ amarillosos o pardos que ocasiona las modificaciones del color. El porcentaje de fermentación muestra una clara tendencia de aumento a medida que avanza la fermentación expresado por su coloración pardo o marrón característico (Barel, 1987).

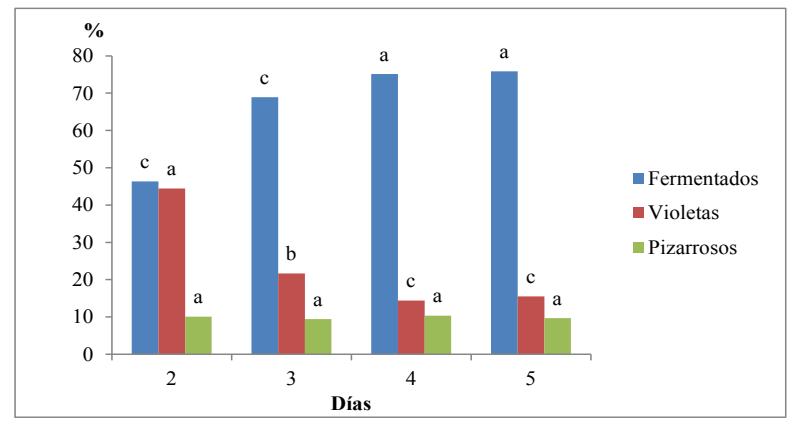

Figura 1. Cambios físicos del grano de cacao en el factor tiempo de fermentación. Medias seguidas por la misma letra no presentan diferencias estadísticas (Tukey, $\mathbf{p}<\mathbf{0 . 0 5}$ ).

Los granos violetas tuvieron un comportamiento inverso a los granos fermentados, es decir que a medida que aumenta el tiempo de fermentación disminuye el porcentaje de granos violeta, ya que se puede observar que el menor contenido de estos granos se encuentra en los últimos días, llegando a reducirse alrededor del $80 \%$ en 5 días de fermentación. Sin embargo, se puede apreciar que dicha reducción tiene mayor pronunciamiento en los primeros tres días, periodo en que se reduce el $70 \%$. Esta disminución reveló un adecuado manejo durante la fermentación, proceso en que las reacciones químicas cambian la coloración de los granos de violeta a marrón (Contreras et al., 2002).

Los granos pizarrosos se mantuvieron alrededor del $10 \%$, y su porcentaje no se vió influenciado por el tiempo de fermentación. Es probable que este porcentaje sea producto de frutos que no tenían un adecuado grado de madurez (Rohan, 1964) al momento de la cosecha. Aunque la normativa ecuatoriana permite entre $4 \mathrm{y}$ $12 \%$ de granos pizarrosos para ciertas clasificaciones, es necesario tomar en cuenta el uso que se le va a dar al cacao, principalmente, si es para la elaboración de chocolates, ya que algunas industrias chocolateras lo asocian con sabores desagradables.

Con respecto a los fermentadores (Figura 2), la caja de madera alcanzó el mayor porcentaje de granos fermentados con $73.3 \%$, en tanto que los demás fermentadores se mantuvieron con un porcentaje inferior al $70 \%$. A diferencia de las variables anteriores y a pesar que registraron diferencias estadísticas significativas (Figura 2) para el factor tipo de fermentador, los porcentaje de granos fermentados y violetas, no mostraron una tendencia clara de aumento o disminución en su contenido. Sin embargo, se observa un mayor porcentaje de granos fermentados y menor porcentaje de granos violetas en la caja de madera. Al igual que el tiempo de fermentación, el tipo de fermentador no influyó en el contenido de granos pizarrosos. La no influencia en esta variable es coherente con la literatura, donde la atribuyen factores como el estado de madurez de la mazorca (Ortiz de Bertorelli et al., 2009), la fermentación deficiente y la falta de volteos, entre otros (Rodríguez, 2006).

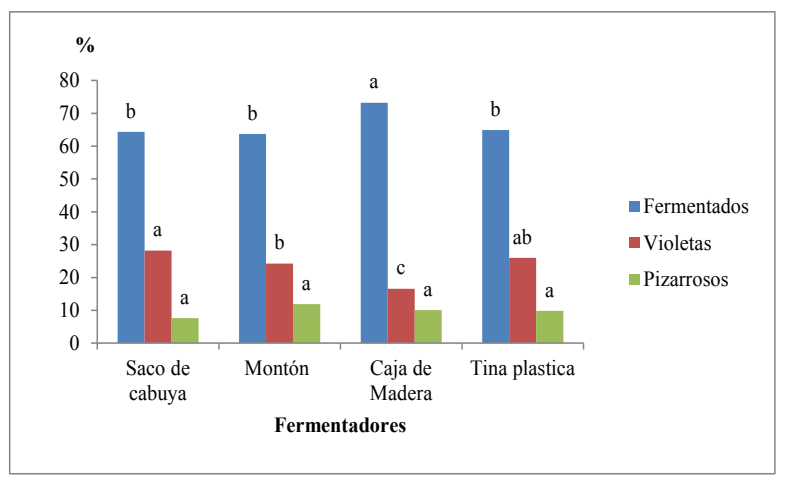

Figura 2. Influencia del tipo de fermentador en los cambios físicos del grano de cacao. Medias seguidas por la misma letra no presentan diferencias estadísticas (Tukey, $\mathbf{p}<\mathbf{0 . 0 5}$ ). 


\section{Variables químicas}

El factor tiempo de fermentación influenció el contenido de polifenoles, presentando diferencias significativas $(\mathrm{p}<0.05)$. El menor contenido se alcanzó al quinto día de fermentación con $38.36 \mathrm{mg} \mathrm{g}^{-1}$, a diferencia del testigo (sin fermentar) que obtuvo el mayor promedio con $98.56 \mathrm{mg} \mathrm{g}^{-1}$ (Figura 3). Estos valores expresan una relación directa entre el tiempo de fermentación y el contenido de polifenoles, y por ende en la calidad organoléptica, ya que al disminuir el contenido de estos compuestos, la astringencia de los granos también disminuye, debido a que esta característica esta relacionada con el sabor desagradable del grano de cacao (Hill y Kold, 1999; Luna et al., 2002). Es necesario mencionar que los polifenoles cobran vital importancia como antioxidantes (Ferrari et al., 2002), por lo que se deben establecer niveles adecuados de estos compuestos que no provoquen una baja calidad sensorial y al mismo tiempo sean beneficiosos para la salud. Con los datos encontrados en esta investigación se podría mencionar que los niveles apropiados podrían fluctuar alrededor de $50 \mathrm{mg} \mathrm{g}^{-1}$. Además, este parámetro podría ser estandarizado entre los requerimientos de calidad exigidos internacionalmente. La variación del contenido de polifenoles se mostró independiente del tipo de fermentador, observándose una tendencia bien definida durante la fermentación, disminuyendo en promedio $61 \%$ al quinto día (Figura 3). Es importante mencionar que el $40 \%$ de la reducción ocurre al segundo día de fermentación con respecto al inicial que estuvo en $98.56 \mathrm{mg} \mathrm{g}^{-1}$. En estudios similares Recalde (2007) encontró una reducción significativa en el primer día.

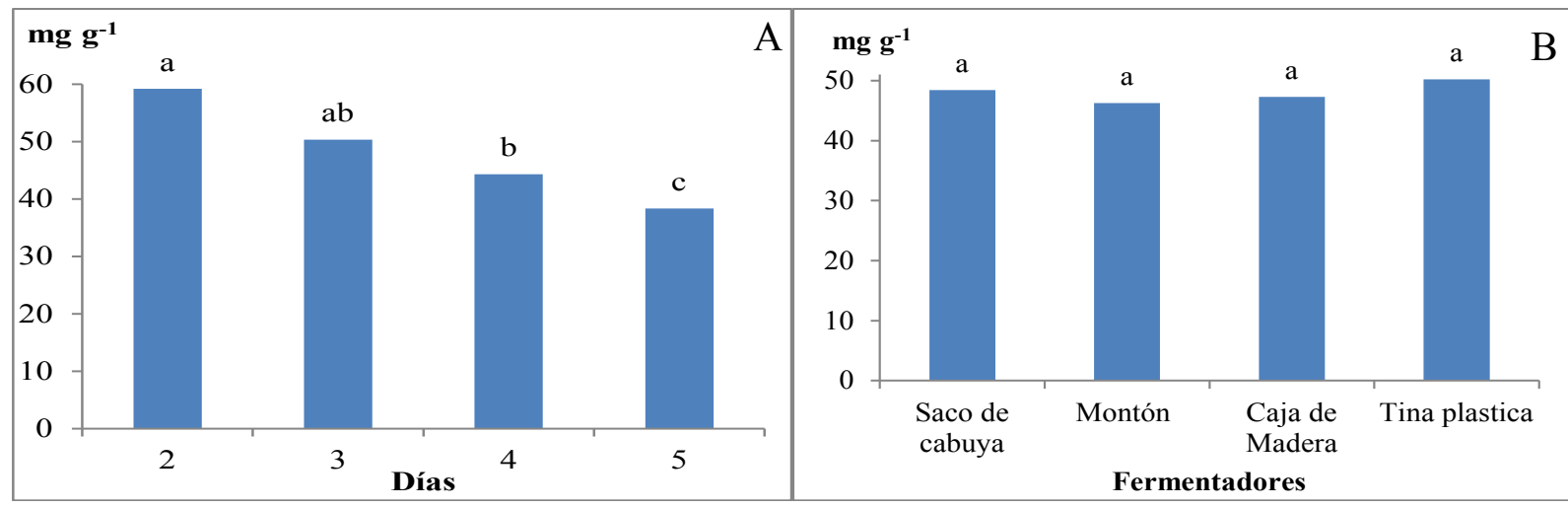

Figura 3. Contenido de polifenoles en el grano de cacao durante el proceso de fermentación, durante cinco días de fermentación (A) y de acuerdo al tipo de fermentadores (B). Medias seguidas por la misma letra no presentan diferencias estadísticas (Tukey, $\mathbf{p}<0.05$ ).

La acidez volátil tuvo un comportamiento variable con el factor tiempo de fermentación, (Figura 4 ), sin embargo, al final se obtuvo un contenido $27 \%$ mayor que el inicial. La reducción de los ácidos volátiles en el tercer día, al no ser inferior a la concentración inicial (en el día cero), sugiere que las reacciones físicas y químicas, responsables de la variación de este compuesto, no ocurrieron en todo el cacao que se sometió al proceso fermentativo, como lo indicó el hecho de que en el análisis físico del grano se encontró siempre un porcentaje de granos sin ningún tipo de cambio.

La acidez volátil esta relacionada sobretodo con el contenido de ácido acético, el cual se origina en la fermentación (Hill y Kold, 1999), además de los ácidos oleico y esteárico (Bruni et al., 2001) que producen aromas agradables.

Al asociar la acidez volátil con la fracción aromática es probable un cambio de los sabores básicos típicos del cacao (acidez, astringencia, amargor, entre otros) a específicos (floral, frutal, nuez, entre otros) característicos del cacao Nacional, sin que represente cambios definidos en su concentración (Hill y Kold, 1999).

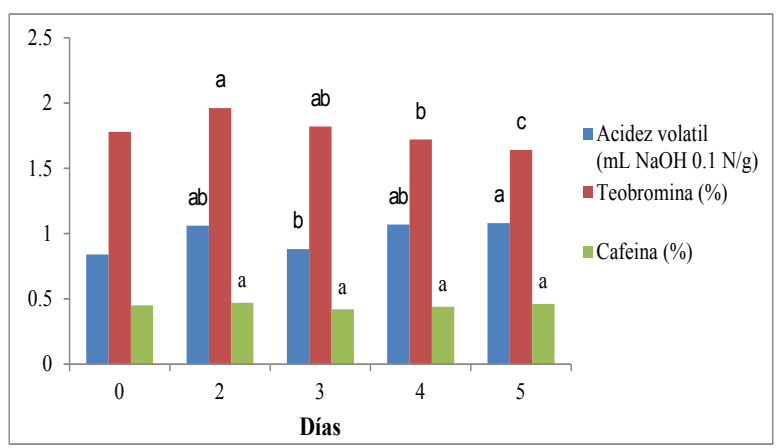

Figura 4. Dinámica de las variables químicas del grano de cacao durante la fermentación. Medias seguidas por la misma letra no presentan diferencias estadísticas (Tukey, $\mathbf{p}<0.05$ ) 
Los alcaloides teobromina y cafeína tuvieron modificaciones leves en su concentración, lo que se reflejó también en el análisis estadístico al sólo encontrar diferencias significativas $(p<0.05)$ en el tiempo de fermentación en la variable teobromina. Este alcaloide se encontró en mayor concentración con respecto a la cafeína (Bucheli et al., 2001). Los alcaloides presentaron ligeras variaciones (Figura 4) con el tiempo de fermentación, alcanzándose al final de la fermentación una reducción promedio de la cafeína de $4.6 \%$ y de $10 \%$ para teobromina. Conjuntamente con los polifenoles, estos alcaloides tiene una correlación positiva al ser responsables de la astringencia y el amargor del cacao (Luna et al., 2002).

\section{Conclusiones}

L os factores en estudio influyeron positivamente sobre la calidad del cacao de tipo Nacional. Los cambios físicos y químicos se inician desde el comienzo de la fermentación con tendencias definidas en la mayoría de las variables analizadas. Se encontraron cambios de las variables químicas estudiadas, más significativos al quinto día de fermentación, siendo la caja de madera la adecuada entre los fermentadores evaluados. A diferencia del tipo de fermentador, el tiempo de fermentación provocó modificaciones físicas y químicas que representaron diferencias estadísticas a excepción de los granos pizarrosos y de la cafeína, observándose que al aumentar los días de fermentación se incrementa, principalmente, el porcentaje de granos fermentados y disminuyen los granos violetas, así como también los polifenoles. Las mejores características de calidad del cacao Nacional se obtuvieron entre los cuatro y cinco días de fermentación en cajas de madera.

\section{Literatura CitADA}

Armijos, A. 2002. Característica de acidez como parámetros químico de calidad en muestras de cacao (Theobroma cacao L) fino y ordinario de producción nacional durante la fermentación. Tesis Dr. Química Pontifica Universidad Católica del Ecuador. Facultad de Ciencia Exactas y Naturales. Quito EC. p.75.

Banco Central del Ecuador (BCE). 2007. Dirección general de estudios: información estadística mensual. Quito. Ecuador. pp. 49-51.

Barel, H. 1987. Délai d écabossage. Influence sur les rendements et la qualité du cacao marchand et du cacao torréfié. Café Cacao Thé 31(2):141-150.

Bruni, R., A. Medici, A. Guerrini, S. Scalia, F. Poli, C. Romagnoli, M. Muzzoli and G. Sacchetti. 2001.
Tocopherol, fatty acids and sterol distributions in wild Ecuadorian Theobroma subincanum (Sterculiaceae). Food Chem. 77:337-341.

Bucheli, P., G. Rousseau, M. Alvarez, M. Laloi and J. McCarthY. 2001. Developmental Variation of Sugars, Carboxylic Acids, Purine Alkaloids, Fatty Acids, and Endoproteinase Activity during Maturation of Theobroma cacao L.Seeds. J. Agric. Food Chem. 49:5046-5051.

Contreras, C., L. Ortiz de Bertorelli, L. Graziani de Fariñas y P. Parra. 2002. Fermentadores para cacao usados por los productores de la localidad de Cumboto, Venezuela. Agron. Trop. 54(2):219232.

Cros, E. and N. Jeanjean. 1995. Cocoa quality: effect of fermentation and drying. Plantations, recherché, développement 24:25-27.

Cros, E., M. Rouly, F. Villeneuve y J. Vicent. 1982. Recherche d' un índice de fermentation du cacao. 11. Estimation de la matiérie colorante rouge des feves de cacao. Café Cacao The 26(2):115-122.

Enríquez, G. 2004. Cacao orgánico: guía para productores ecuatorianos. Quito, Ecuador. p. 360.

Espin, S., L. Samaniego, H. Wakao, J. Jimenez. 2007. La relación Teobromina/cafeína asociada a la calidad de cacao ecuatoriano. Memorias del VI Congreso Iberoamericano de Ingeniería en Alimentos. Riobamba, EC. pp. 107-109.

Ferrari, C. K. B. and E. A. F. S. Torres. 2002. Biochemical pharmacology of functional foods and prevention of chronic diseases of aging. Biomedicine \& Pharmacotherapy 57:251-260.

Graziani de Fariñas, L., L. Ortiz de Bertorelli, J., N. Álvarez y A. Trujillo de Leal. 2003. Fermentación del cacao en dos diseños de cajas de madera. Agron. Trop. 53(2):175-187.

Hill, J. W. y D. K. Kolb. 1999. Química para el nuevo milenio. México $.8^{\circ}$ ed. p. 704.

Luna, F., D. Crouzillat, L. Cirou, and P. Bucheli. 2002. Chemical Composition and Flavor of Ecuadorian Cocoa Liquor. J. Agrid. Food Chem. 50:35273532.

Manual de Productos Básicos. 1991. Cacao fino de aroma. Estudio de la producción y el comercio mundial. Centro de Comercio Interno. UNCTAD/ GATT. Ginebra. p. 60.

Ortiz de Bertorelli, L., L. Graziani de Fariñas y L. Gervaise. 2009. Influencia de varios factores sobre características del grano de cacao fermentado y secado al sol. Agron. Trop. 59(2):119-127.

Puziah, H., S. Jianp, M. Kharidah and A. Asbi. 1998. Effect of mass and turning time on free amino acid, peotide-N, sugar and pyrazine concentration 
Rivera et al.

during cocoa fermentation. J. Sci. Food Agric. Tomlins, K., D. Baker, P. Daplyn and D. Adomako. 78:543-550.

Recalde, A. 2007. Evaluación del efecto del procesado y tiempo de la fermentación en los contenidos de Polifenoles, alcaloides y ácidos volátiles en dos genotipos de cacao. Tesis Dr. Químico. Universidad Central del Ecuador. Facultad de Ciencias Químicas. Quito, EC. p. 79.

Rohan, T. 1964. El beneficio del cacao bruto destinado al mercado. Organización de las Naciones Unidas para la Agricultura y la Alimentación. Roma, Italia. p. 223.

Rodríguez, N. Beneficio del cacao. Facultad de agronomía de U. C. V. Departamento e Instituto de Agronomía. VE. p. 25-27. 1993. Effect of fermentation and drying practies on the chemical and physical profiles of Ghana cocoa. Food Chem. 46:257-263.

Torres, O., L. Graziani de Fariñas, L. Ortiz de Bertorelli y A. Trujillo. 2004. Efecto del tiempo transcurrido entre la cosecha y el desgrane de la mazorca del cacao tipo forastero de Cuyagua sobre características del grano en fermentación. Agron. Trop. 54(4):481-495. 